\title{
Gut microbiota variations in a rescued spotted seal Phoca largha pup
}

\author{
Jiashen Tian, Jing Du, Jiabo Han, Zhen Wang, Zhiyu Fu, Zhichuang Lu* \\ Dalian Key Laboratory of Conservation Biology for Endangered Marine mammals, Liaoning Ocean and \\ Fisheries Science Research Institute, 50 Heishijiao Road, Shahekou District, Dalian, Liaoning 116023, PR China
}

\begin{abstract}
Rescuing seal pups is an important conservation action for spotted seals Phoca largha. Gut microbiota are directly associated with host health and diet metabolism. Therefore, knowledge gained from gut microbiota variations of spotted seal pups held in captivity after rescue can help formulate comprehensive rescue plans for the future. In this study, we collected feces from a rescued spotted seal pup every $3 \mathrm{~d}$ during the rescue process. Fecal bacterial communities were measured by high-throughput sequencing based on 16S rRNA amplification. Firmicutes were the most dominant bacteria, comprising $>70 \%$ of the total gut microbiota. Moreover, differences in fecal bacterial communities of the rescued spotted seal pup between rescue and release were compared. At release, the abundances of 2 potential bacteria related to gut health, Blautia producta and Cetobacterium somerae, were remarkably lower, while Clostridium perfringens, a key mammalian pathogen, was significantly higher in the feces of the released pup. Moreover, the pup experienced a bout of diarrhea during its time in captivity, which resulted in a momentary change in its gut microbiota. Fusobacterium was recognized as a potential causative pathogen for the diarrhea. This study contributes to our understanding of gut microbiota variations in spotted seal pups during the rescue period.
\end{abstract}

KEY WORDS: Spotted seal $\cdot$ Phoca larga $\cdot$ Gut microbiota $\cdot$ Diarrhea $\cdot$ Rescue $\cdot$ Clostridium perfringens $\cdot$ Fusobacterium

\section{INTRODUCTION}

Between November and May, spotted seals Phoca largha assemble in large groups in the Liaodong Gulf of China to mate and reproduce (Wang 1986). Currently, the $P$. largha population at this locality is estimated at approximately 2000 ; thus, these animals are categorized as grade-II endangered species in China (Gao et al. 2015). The simultaneous occurrence of onice births, weaning pups, and ice-melting are important factors in the reproductive biology of these seals (Atkinson 1997). Increased ice-melting due to global warming and harsh sea conditions have caused high death rates of spotted seal pups (Zhang et al. 2014); therefore, it is important to increase the survival rate of pups by rescuing animals in need.

\footnotetext{
*Corresponding author: luzhichuang@hotmail.com
}

Environment and diet influence pinniped gut microbiota (Nelson et al. 2013). For example, environmental microbes from sea water at different colony locations led to differences in gut bacteria in sea lions (Delport et al. 2016). Moreover, different prey have been shown to contribute to gut microbiota differences between wild and captive leopard seals (Nelson et al. 2013). During a rescue process, the environment of a spotted seal pup invariably changes from a natural to an artificial one, requiring a modified diet and impacting gut microbiota.

In this study, gut microbiota variations in a rescued spotted seal pup were evaluated by high-throughput sequencing of 16S rRNA genes. The pup suffered from diarrhea while within captivity; however, the

() The authors 2020. Open Access under Creative Commons by Attribution Licence. Use, distribution and reproduction are unrestricted. Authors and original publication must be credited. 
condition was cured before release. Our study objectives were to explore differences in gut microbiota between rescue and release, and identify the potential cause of the diarrhea. To the best of our knowledge, this study is the first to analyze changes in the gut microbiota of a spotted seal during the rescue period.

\section{MATERIALS AND METHODS}

\subsection{Rescue period}

A spotted seal pup (weight: $23.0 \mathrm{~kg}$, body length: $87 \mathrm{~cm})$ was found in Liaodong Gulf $\left(121.56^{\circ} \mathrm{N}\right.$, $40.87^{\circ} \mathrm{E}$ ) on 4 March 2019 on a small piece of sea ice, separated from its mother. Based on white lanugo on its skin and the fact that it was away from the mother, the age of the pup was inferred at $<1$ mo. During this lactation period, pups cannot hunt by themselves; therefore, this pup was rescued to ensure its survival. During the rescue period, the pup was housed in a $3 \times 5 \times 1.5 \mathrm{~m}$ aquarium, located in Liaoning Ocean and Fisheries Science Research Institute. The pup was fed Atlantic herring Clupea harengus at a rate of $10 \%$ of its body weight every day. Vitamin B in fish tissue rapidly breaks down after death, which can lead to anorexia in seal pups; therefore, multi-vitamins/multi-minerals were added to supplement the fish diet at a dose of 1 tablet $d^{-1}$, to ensure normal growth. On Day 6 after capture, the pup experienced diarrhea, with loose and watery stool during bowel movements. Multi-enzyme tablets were administered to treat the condition. Diarrhea symptoms subsided on Day 12. The entire rescue period lasted $24 \mathrm{~d}$.

\subsection{Fecal collection, DNA sequencing, and bioinformatics analyses}

During the rescue period, 3 fecal samples were collected in sterilized containers every $3 \mathrm{~d}$. After collection, the surface part of each fecal sample that had been in contact with the water was removed, and only the inside, uncontaminated material was reserved. In total, 27 fecal samples were collected. Bacterial genomic DNA was extracted using the QIAamp PowerFecal DNA Kit (QIAGEN). Sequencing and bacterial community annotation were performed according to a previous study (Zhao et al. 2018). Briefly, the V3-V4 region of the 16S rRNA gene was amplified by PCR, and amplicon libraries were generated after purification, quantification, and equiva- lent mixing of amplification products. The libraries were sequenced on an Illumina MiSeq platform with a 250 bp paired-end strategy. After quality control, clean reads were assembled into tags, clustered into operational taxonomic units, and taxonomically annotated by FLASH, QIIME, and Greengenes, respectively.

\subsection{Statistical analysis}

Principal coordinate analysis was performed by the 'vegan' (Oksanen et al. 2019) and 'ggplot2' (Wickham 2009) packages in the R platform (R Core Team 2012) to show variations in gut microbiota composition during the rescue period. The relative abundance of dominant bacteria at the phylum level was revealed by the 'ggalluvial' (Brunson 2019) package in R. Differences in the relative abundance of bacteria at each bacterial species level between the initial rescue and release were evaluated by $t$-tests and their relative abundances were investigated by the 'ggplot2' package in R. Significant difference in the relative abundance of a bacterial species between the initial rescue and release was confirmed if $\mathrm{p}<$ 0.05 .

\section{RESULTS AND DISCUSSION}

\subsection{Variations in gut microbiota during the rescue period}

The spotted seal pup's fecal microbial community composition on Day 3 was similar to before the initial rescue (Fig. 1a). Shifts in gut microbiota were observed on Day 6, in line with the appearance of diarrhea (Fig. 1a). More significant alterations in gut microbiota were observed in fecal samples collected on Days 9 and 12 (Fig. 1a). After the diarrhea was treated, differences in gut microbiota gradually diminished between Days 15 and 21 (Fig. 1a). Finally, gut microbiota recovered to the state of initial rescue (Fig. 1a). These data show that the diarrhea episode was associated with a change in gut microbiota; however, these changes were eventually restored.

Firmicutes were the dominant bacteria $(74.2 \%)$ in the gut microbiota, followed by Fusobacteria, Proteobacteria, Actinobacteria, and Bacteroidetes, which accounted for 15.3, 4.9, 2.7, and $2.6 \%$ of the total gut microbiota, respectively (Fig. 1b). Firmicutes are also dominant bacteria in the gut microbiota of other pinnipeds, including Australian sea 


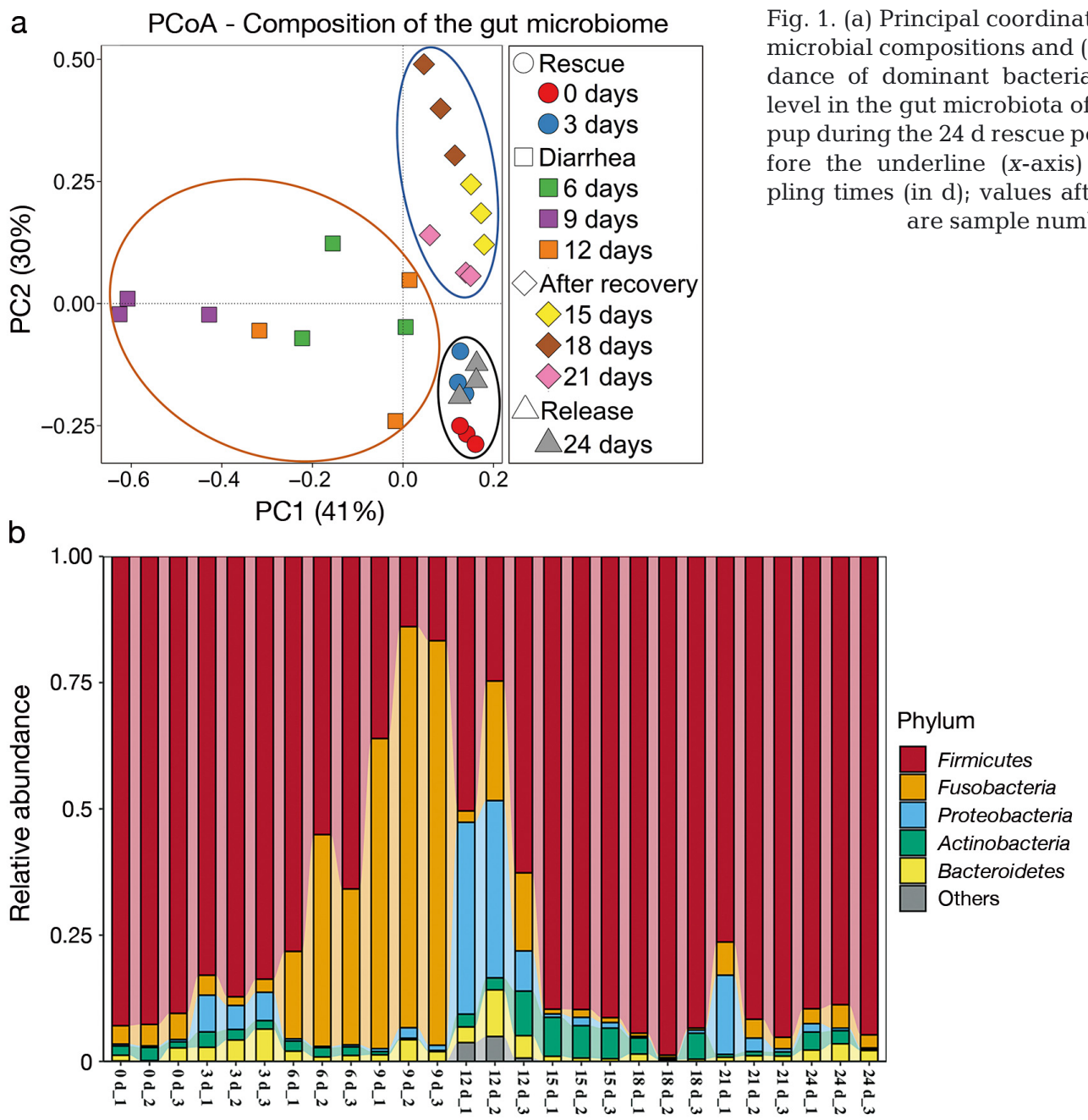

lions (Siebert et al. 2009), fur seals (Smith et al. 2013), and Antarctic seals (Nelson et al. 2013). Firmicutes enrichment in animal guts can lead to more efficient energy absorption from food (Estrada-Velasco et al. 2015). In humans and mice, the relative ratio of Firmicutes to Bacteroidetes is a factor in obesity, with obese humans and mice having relatively fewer Bacteroidetes, but more Firmicutes, when compared to leaner counterparts (Ley et al. 2006). Spotted seals have a high body fat content to ensure survival in cold seawater environments (Jefferson et al. 2007); thus, high Firmicutes abundance in the guts of these animals could be related to fat production.

\subsection{Differences in gut microbiota between rescue and release}

Although the gut microbiota of the pup at release were similar to when it was initially rescued, we ob- served significant changes in the relative abundance of some microorganisms (Fig. 2a). When compared to microbiota levels at the time of rescue, the relative abundance of Clostridium perfringens was significantly higher at release ( $t$-test, $\mathrm{p}<0.05$; Fig. 2a). $C$. perfringens is a widely distributed pathogen found in the intestinal flora of most animals and humans (Morris \& Fernández-Miyakawa 2009). It is possible these increased $C$. perfringens levels in the gut microbiota may have increased disease risks for the pup following release.

In contrast to $C$. perfringens, the relative abundances of Blautia producta and Cetobacterium somerae were significantly lower in the pup's gut microbiota at release compared to initial rescue ( $t$-test, p $<0.05 ;$ Fig. 2a). These gut bacteria are beneficial to host health; $B$. producta clears vancomycin-resistant Enterococcus from mouse intestines, inhibiting bloodstream infections (Caballero et al. 2017), while C. somerae produces acetic acid as 

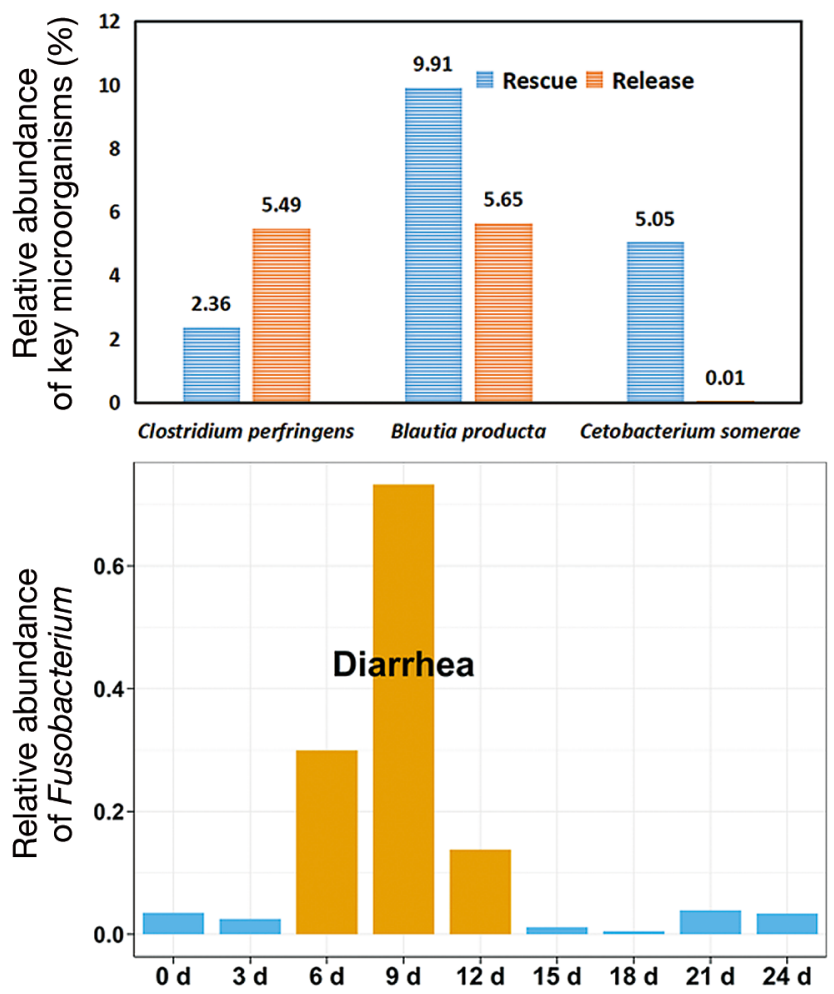

Fig. 2. (a) Bacteria in spotted seal pup feces showing significant differences in abundance between rescue and release. (b) Relative abundance of Fusobacterium in the gut microbiota during the rescue period

a major end product of peptide and carbohydrate metabolism (Finegold et al. 2003). G protein-coupled receptor 43 (GPR43) activation by acetic acid exerts significant protective effects on mouse intestinal inflammation (Sivaprakasam et al. 2017). Accordingly, these gut bacteria may benefit seal pup health; however, as these bacterial levels were lower than when the pup was initially rescued, this may have reflected poor pup gut health at release.

\subsection{Variations in gut microbiota during diarrhea}

We analyzed shifts in dominant bacteria abundance in the pup gut to identify potential reasons for diarrhea during the rescue period. During the diarrheal stage, the relative abundance of Fusobacteria was significantly higher than in other non-diarrheal fecal samples (Fig. 1b) and largely comprised members of the Fusobacterium genus (Fig. 2b). Fusobacterium is a Gram-negative, non-spore-forming bacterium, widely accepted as a human and animal pathogen (Castellarin et al. 2012). Significantly higher Fusobacterium levels have been detected in oral-type tissues from diseased striped dolphins compared with healthy counterparts (Godoy-Vitorino et al. 2017). Thus, diarrhea in our rescue pup may have occurred from unusual Fusobacterium enrichment in its gut. Unfortunately, this Fusobacterium was not classified to the species level. The phylogeny and pathogenic mechanisms of this bacteria should be studied to develop effective methods to prevent potentially debilitating diarrhea in rescued animals.

\section{CONCLUSIONS}

Firmicutes was the most dominant bacteria in the rescued seal pup's feces. The overall composition of gut microbiota was similar at the initial capture and release. However, we observed significant changes in the relative abundance of some bacteria before and after rescue. Amounts of Clostridium perfringens were higher, while levels of Blautia producta and Cetobacterium somerae were lower at release. Moreover, diarrhea occurred during the rescue period, after which a brief shift in gut microbiota composition was observed; Fusobacterium appeared to be related to the occurrence of diarrhea in this animal.

Acknowledgements. This work was supported by the Foundation of Liaoning Province, Department of Ocean and Fisheries (201812/201822), and China Environment and Zoology Protection for Offshore Oil and Ocean Foundation (CFMEEC/ER/2019-10). All international, national, and/or institutional guidelines for the care and use of animals were followed. The rescue of the spotted seal pup was authorized by the Ministry of Agriculture and Rural Affairs of the People's Republic of China (permit number: 1376).

\section{LITERATURE CITED}

Atkinson S (1997) Reproductive biology of seals. Rev Reprod 2:175-194

* Brunson JC (2019) ggalluvial: Alluvial plots in 'ggplot2'. https://CRAN.R-project.org/package=ggalluvial

Caballero S, Kim S, Carter RA, Leiner IM, Pamer EG (2017) Cooperating commensals restore colonization resistance to vancomycin-resistant Enterococcus faecium. Cell Host Microbe 21:592-602

Castellarin M, Warren RL, Freeman JD, Dreolini L and others (2012) Fusobacterium nucleatum infection is prevalent in human colorectal carcinoma. Genome Res 22: 299-306

* Delport TC, Power ML, Harcourt RG, Webster KN, Tetu SG (2016) Colony location and captivity influence the gut microbial community composition of the Australian sea lion (Neophoca cinerea). Appl Environ Microbiol 82: 3440-3449

*Estrada-Velasco BI, Cruz M, Garcia-Mena J, Valladares Salgado A and others (2015) Childhood obesity is associated to the interaction between Firmicutes and high energy 
food consumption. Nutr Hosp 31:1074-1081

Finegold SM, Vaisanen ML, Molitoris DR, Tomzynski TJ and others (2003) Cetobacterium somerae sp. nov. from human feces and emended description of the genus Cetobacterium. Syst Appl Microbiol 26:177-181

Gao XG, Han JB, Lu ZC, Zhang PJ, He CB (2015) Short communication sequence variation and gene duplication at the MHC DRB loci of the spotted seal Phoca largha. Genet Mol Res 14:2055-2062

Godoy-Vitorino F, Rodriguez-Hilario A, Alves AL, Gonçalves F and others (2017) The microbiome of a striped dolphin (Stenella coeruleoalba) stranded in Portugal. Res Microbiol 168:85-93

Jefferson TA, Webber MA, Pitman RL (2007) Marine mammals of the world: a comprehensive guide to their identification. Academic Press, London

Key RE, Turnbaugh PJ, Klein S, Gordon JI (2006) Microbial ecology: human gut microbes associated with obesity. Nature 444:1022-1023

Morris WE, Fernández-Miyakawa ME (2009) Toxins of Clostridium perfringens. Rev Argent Microbiol 41: 251-260

Nelson TM, Rogers TL, Carlini AR, Brown MV (2013) Diet and phylogeny shape the gut microbiota of Antarctic seals: a comparison of wild and captive animals. Environ Microbiol 15:1132-1145

Oksanen J, Blanchet FG, Friendly M, Kindt R and others (2019) vegan: community ecology package. R package version 2.5-6. https://CRAN.R-project.org/package=vegan

Editorial responsibility: Victor Benno Meyer-Rochow, Oulu, Finland
R Core Team (2012) R: a language and environment for statistical computing. R Foundation for Statistical Computing, Vienna

Siebert U, Prenger-Berninghoff E, Weiss R (2009) Regional differences in bacteria flora in harbour porpoises from the North Atlantic: environmental effects. J Appl Microbiol 106:329-337

Sivaprakasam S, Bhutia YD, Ramachandran S, Ganapathy V (2017) Cell-surface and nuclear receptors in the colon as targets for bacterial metabolites and its relevance to colon health. Nutrients 9:856

* Smith SC, Chalker A, Dewar ML, Arnould JP (2013) Agerelated differences revealed in Australian fur seal Arctocephalus pusillus doriferus, gut microbiota. FEMS Microbiol Ecol 86:246-255

Wang P (1986) Distribution, ecology and resource conservation of the spotted seal in the Huanghai and Bohai Seas. Acta Oceanol Sin 1:1-14

Wickham H (2009) ggplot2: Elegant graphics for data analysis. Springer, New York, NY. https://CRAN.R-project.org/ package $=$ ggplot 2

* Zhang PJ, Song XR, Han JB, Wang LM, Yang Y (2014) Milk composition, milk consumption, and growth rate of a captive spotted seal (Phoca largha) pup from Liaodong Bay, China. Can J Zool 92:449-452

Z Zhao Z, Pan Y, Jiang J, Gao S and others (2018) Unrevealing variation of microbial communities and correlation with environmental variables in a full culture-cycle of Undaria pinnatifida. Mar Environ Res 139:46-56

Submitted: March 30, 2020; Accepted: May 19, 2020

Proofs received from author(s): July 5, 2020 